\title{
Sur la construction asymptotique et l'interprétation physique d'une théorie avec dégénérescence du vide
}

\author{
A. N. VASSILEV \\ l'Université de Leningrade, USSR
}

Reçu le 6 Fevrier 1969

\begin{abstract}
The study of the set Q of Wightman's functionals begun in ref. [5] is continued in this paper. Haag-Ruelle asymptotic construction $[1,2]$ is formulated for the case when all the pure Wighтмах's functionals contained in the decomposition of the given functional $\bar{w} \in Q$ generate the same set of asymptotic states. As an example we consider a theory with the degenerate vacuum and prove that it is physically equivalent to a theory with the single vacuum. For this case we show that the transition to the theory with the degenerate vacuum is equivalent to introducing of a charged spurion in the theory with the single vacuum. The mathematically correct creation and destruction operators for this spurion are given.
\end{abstract}

\section{Introduction}

Ce travail est le prolongement direct de l'article [5] dont les résultats et les notations seront utilisés sans explication supplémentaire.

Nous étudierons la construction asymptotique de HAAG-RUElLE $[1,2]$ pour les fonctionelles de la classe $Q$ [5], autrement dit, pour des fonctionelles de la forme:

$$
\bar{w}=-\int_{s} w d \mu(w)
$$

où $s$ est un ensemble faiblement compact des fonctionelles de la classe $Q_{0}$ et $\mu$ une mesure sur $s$ normée à l'unité. Par définition la classe $Q_{0}$ est constituée par toutes les fonctionelles de Wightman satisfaisant à la condition spectrale forte et à celle d'unicité du vide [5]. Les fonctionelles de la classe $Q$ engendrent les théories avec dégénérescence du vide.

D'habitude en formulant les axiomes de la théorie quantique des champs on y ajoute la condition d'unicité du vide en supposant sans l'avoir dit explicitement que la théorie avec dégénérescence du vide ne peut pas correspondre à la réalité.

Pour se faire une idée sur ce sujet considérons le cas le plus simple de la dégénérescence du vide - la théorie engendrée par la fonctionelle $\bar{w}$ :

$$
\bar{w}=\varrho_{1} w_{1}+\varrho_{2} w_{2} \varrho_{1,2}>0 \varrho_{1}+\varrho_{2}=1 \quad w_{1} \neq w_{2} w_{1,2} \in Q_{0} .
$$

L'espace hilbertien de cette théorie est la somme directe des sous-espaces $H_{w_{1}}$ et $H_{w_{2}}$ engendrés par $w_{1}, w_{2}$ respectivement, le sous-espace de vide [5] est de dimension 2. 
En appliquant la construction asymptotique de Haag-Ruelle aux fonctionelles $w_{1,2}$ (nous supposons naturellement que c'est possible) construisons les champs asymptotiques $A_{w_{1,2}}^{\text {in(out) }}(x)$ dans $H_{u_{1,2}}$ respectivement. Soient $\left\{m_{\alpha}^{i}, s_{\alpha}^{i}\right\} i=1,2 \alpha=1,2, \ldots$ les caractéristiques des particules asymptotiques $\left(m_{\alpha}\right.$ désigne la masse de la particule numéro $\alpha$, $s_{\alpha}$ - d'autres nombres quantiques de cette particule) des fonctionelles $w_{i} i=1,2$ respectivement.

Considérons successivement différentes possibilités.

1. Un des ensembles, par exemple, $\left\{m_{\alpha}^{1}, s_{\alpha}^{1}\right\}$ est vide. Autrement dit, la fonctionelle $w_{1}$ n'engendre aucune particule asymptotique. Dans ce cas on peut traiter le problème sans faire intervenir la fonctionelle $w_{1}$ parce que cette dernière n'influence pas les amplitudes de transition des particules asymptotiques qui sont les seuls objets mesurables.

2. Les deux ensembles $\left\{m_{\alpha}^{i}, s_{\alpha}^{i}\right\} i=1,2$ étant non vides, tout ensemble contient une particule n'ayant pas d'analogue dans l'autre ensemble. Soient $a_{i}^{+}(p)$ les opérateurs de création de ces particules définis dans $H_{u_{i}} i=1,2$ respectivement.

Si de telles particules existaient elles auraient une propriété étrange: on ne pourrait jamais les observer simultanément. En effet dans une semblable théorie les seuls états contenant explicitement les opérateurs de création des particules de deux sortes sont des superpositions

$$
a_{1}^{+}\left(p_{1}\right) \ldots a_{1}^{+}\left(p_{n}\right) \psi_{w_{1}}(1)+a_{2}^{+}\left(q_{1}\right) \ldots a_{2}^{+}\left(q_{m}\right) \psi_{w_{2}}(1)
$$

où $\psi_{w_{1,2}}(1)$ sont des vides dans $H_{w_{1,2}}$ respectivement. Mais ce n'est pas ce qu'il faut car d'après l'interprétation classique de la mécanique quantique une telle superposition décrit un état tel qu'un observateur peut trouver ou bien les $n$ particules de la première sorte ou bien les $m$ particules de la deuxième sorte mais jamais les particules de deux sortes à la fois.

Etant donné que dans la nature il n'y a pas de telles particules une théorie de ce genre doit être rejetée.

3. Les deux ensembles $\left\{m_{\alpha}^{i}, s_{\alpha}^{i}\right\} i=1,2$ sont identiques, par exemple, $m_{\alpha}^{1}=m_{\alpha}^{2}, s_{\alpha}^{1}=s_{\alpha}^{2}$ pour tout $\alpha=1,2, \ldots$ Désignons par $a_{i \alpha}^{+}(p)$ les opérateurs de création des particules du type $\alpha$ dans $H_{w_{i}} i=1,2$ respectivement.

Cette fois on doit choisir entre deux interprétations possibles. L'une d'elles consiste à interpréter $a_{1 \alpha}^{+}(p)$ et $a_{2 \alpha}^{+}(p)$ comme les opérateurs de création des particules différentes en entendant par cela que les deux états $a_{1 \alpha}^{+}(p) \psi_{w_{1}}(1)$ et $a_{2 \alpha}^{+}(p) \psi_{w_{2}}(1)$ peuvent être distingués expérimentalement.

Il est clair que cette interprétation se heurte aux mêmes difficultés que la théorie considérée ci-dessus (le cas numéro 2). 
Une autre interprétation consiste à postuler qu'il existe une seule particule du type $\alpha$ et à prendre pour opérateur de création de cette particule la somme directe:

$$
a_{\alpha}^{+}(p)=a_{1 \alpha}^{+}(p) \oplus a_{2 \alpha}^{+}(p) \equiv\left(\begin{array}{cc}
a_{1 \alpha}^{+}(p) & 0 \\
0 & a_{2 \alpha}^{+}(p)
\end{array}\right)
$$

et de même pour l'opérateur de destruction. Les opérateurs de création et de destruction définis de cette façon satisfont évidemment aux relations de commutation canoniques.

Dans ce cas nous avons donc à expliquer en quoi consiste la différence entre les états $a_{1 \alpha}^{+}(p) \psi_{w_{1}}(1)$ et $a_{2 \alpha}^{+}(p) \psi_{w_{2}}(1)$. Nous disons que ces deux états ne peuvent pas être distingués expérimentalement et représentent la même particule excitée au-dessus des vides différents:

$$
a_{\alpha}^{+}(p)\left(\begin{array}{c}
\psi_{w_{1}}(\mathrm{I}) \\
0
\end{array}\right)=\left(\begin{array}{c}
a_{1 \alpha}^{+}(p) \psi_{w_{1}}(\mathrm{I}) \\
0
\end{array}\right) \quad a_{\alpha}^{+}(p)\left(\begin{array}{c}
0 \\
\psi_{w_{2}}(\mathrm{I})
\end{array}\right)=\left(\begin{array}{c}
0 \\
a_{2 \alpha}^{+}(p) \psi_{w_{2}}(\mathrm{I})
\end{array}\right)
$$

et de même pour des états contenant plus qu'une particule.

Comme une telle théorie n'est pas apparemment en contradiction avec l'expérience, nous l'étudions en détail dans les paragraphes suivants.

4. Toute particule d'un ensemble, par exemple, de $\left\{m_{\alpha}^{1}, s_{\alpha}^{1}\right\}$ a unc analogue dans l'autre mais la réciproque est inexacte. Alors les particules de $\left\{m_{\alpha}^{2}, s_{\alpha}^{2}\right\}$ se divisent en deux groupes: les particules ayant une analogue dans $\left\{m_{\alpha}^{1}, s_{\alpha}^{1}\right\}$ et celles qui n'en ont pas. Si l'on adopte pour les particules du premier groupe la description ci-dessus, une telle théorie pourrait être raisonable mais nous ne la considérons pas dans cet article.

Nous avons examiné toutes les situations possibles dans le cas où la dégénérescence du vide est de dimension 2 . Il est clair que l'essentiel de cet exposè ne change pas lorsqu'on passe au cas de la dégénérescence plus puissante.

Mais il faut tenir compte que la forme de la dégénérescence du vide dont nous avons parlé ci-dessus n'est pas la seule possible. Nous n'avons considéré que la dégénérescence provenant de la réductibilité du champ alors que, comme on le sait [3], le vide peut être dégénéré même si le champ est irréductible. Cette dernière forme de la dégénérescence reste hors de notre considération car nous nous bornons aux fonctionelles de la classe $Q$ pour lesquelles nous connaissons bien la structure algébrique de la représentation [5].

\section{L'espace asymptotique}

Soient $\bar{w}$ une fonctionelle de la classe $Q$ :

$$
\bar{w}=\int_{s} w d \mu(w)
$$

$R_{\bar{w}}$ - la représentation correspondante dans l'espace hilbertien $H_{\bar{w}}[5]$. 


\section{Supposons que:}

1. toute fonctionelle $w \in s$ est telle qu'il est possible de construire par le procédé de HaAG-Ruelce les champs asymptotiques $A_{w}^{\text {in (out) }}(x)$ et la matrice $S_{w}$ :

$$
A_{w}^{\text {out }}(x)=S_{w} A_{w}^{\text {in }}(x) S_{w}^{-1}
$$

(dans toute la suite de ce paragraphe nous dirons «le champ asymptotique» et écrivons $A_{w}^{\text {in (out) }}(x)$ même s'il s'agit de tout un système de champs),

2 . pour toute $w \in s$ la matrice $S_{w}$ est un opérateur unitaire, autrement dit [2], les sous-espaces asymptotiques $H_{w}^{\text {in (out) }}$ [les sous-espaces engendrès à partir du vide $\psi_{w}(1)$ par les champs $A_{w}^{\text {in (out) }}(x)$ respectivement] coincident. Notons:

$$
\begin{gathered}
H_{w}^{\text {as }} \equiv H_{w}^{\text {in }}=H_{w}^{\text {out }} \leqq H_{w} \\
r_{w}^{\text {in (out) }}(a) \equiv \sum_{n} \int \cdots \int d x_{1}, \ldots d x_{n} a_{n}\left(x_{1} \cdots x_{n}\right) A_{w}^{\text {in }(\text { out })}\left(x_{1}\right) \ldots A_{w}^{\text {in (out })}\left(x_{n}\right) \\
\psi_{w}^{\text {in (out) }}(a) \equiv r_{w}^{\text {in (out) }}(a) \psi_{w}(1), w^{\text {as }}(a) \equiv\left\langle\psi_{w}(1), \psi_{w}^{\text {in }}(a)\right\rangle=\left\langle\psi_{w}(1), \psi_{w}^{\text {out }}(a)\right\rangle .
\end{gathered}
$$

Supposons de plus que:

3. la fonctionelle asymptotique est la même pour toute $w \in s$ :

$$
w_{1}^{\mathrm{as}}=w_{2}^{\mathrm{as}} \forall w_{1,2} \in s .
$$

La dernière condition signifie que toutes les fonctionelles de $s$ engendrent des systèmes identiques de particules asymptotiques. L'analyse du paragraphe précédent explique pourquoi nous nous intéressons à ce cas particulier.

Notons:

$$
H_{\bar{w}}^{\mathrm{as}} \equiv \int_{s} \oplus H_{w}^{\mathrm{as}} d \mu(w) \leqq \int_{s} \oplus H_{w} d \mu(w) \equiv H_{\bar{w}}
$$

Définissons les champs asymptotiques dans $H_{\bar{w}}^{\text {as }}$ par les intégrales directes:

Evidemment

$$
A_{\bar{w}}^{\text {in (out) }}(x)=\int_{s} \oplus A_{w}^{\text {in (out) }}(x) .
$$

$$
A_{\bar{w}}^{\text {out }}(x)=S_{\bar{w}} A_{\bar{w}}^{\text {in }}(x) S_{\bar{w}}^{-1} \quad \text { où } \quad S_{\bar{w}}=\int_{s} \oplus S_{w}
$$

est un opérateur unitaire dans $H_{\bar{w}}^{a s}$.

Il résulte de la définition de $H_{\bar{w}}^{\text {as }}$ qu'il contient tout le sous-espace de vide $H \frac{0}{w}$ :

Désignons :

$$
H_{\bar{w}}^{0}=\left\{\psi_{\bar{w}}(1, f): f \in L_{2}(\mu, s)\right\} \subset H_{\bar{w}}^{\mathrm{as}} .
$$

$$
\psi_{\bar{w}}^{\text {in }}{ }^{\text {out })}(a, f) \equiv r_{\bar{w}}^{\text {in }}{ }^{\text {out })}(a) \psi_{\bar{w}}(1, f)
$$

et par $H_{\bar{w}}^{\text {in (out) }}(f)$ la fermeture de l'ensemble $\left\{\psi_{\bar{w}}^{\text {in (out) }}(a, f): a \in A\right\}$ dans la norme de $H_{\bar{w}}$. 
Proposition 1. Soient $f_{1,2}$ deux fonctions orthogonales dans $L_{2}(\mu, s)$. Les sous-espaces $H_{\bar{w}}^{\text {in }}$ (out) $\left(f_{1}, 2\right)$ sont alors orthogonaux. Pour toute base orthonormale $f_{n} n=1,2, \ldots$ dans $L_{2}(\mu, s)$

$$
H_{\bar{w}}^{\text {as }}=\sum_{n=1}^{\infty} \oplus H_{\bar{w}}^{\mathrm{in}}\left(f_{n}\right)=\sum_{n=1}^{\infty} \oplus H_{\bar{w}}^{\text {out }}\left(f_{n}\right) .
$$

Démonstration. On a:

$$
\begin{aligned}
\left\langle\psi_{\bar{w}}^{\text {in (out) }}\left(a, f_{1}\right),\right. & \left.\psi_{\bar{w}}^{\text {in (out) }}\left(b, f_{2}\right)\right\rangle \\
& =\int_{s}\left\langle\psi_{w}^{\text {in (out) }}(a), \psi_{w}^{\text {in out })}(b)\right\rangle f_{1}^{*}(w) f_{2}(w) d \mu(w) \\
& =\int_{s} w^{\text {as }}\left(a^{+} b\right) f_{1}^{*}(w) f_{2}(w) d \mu(w) \\
& =w^{\text {as }}\left(a^{+} b\right) \int_{s} f_{1}^{*}(w) f_{2}(w) d \mu(w)
\end{aligned}
$$

d'où la première assertion de la proposition.

Par définition tout vecteur $\psi_{\bar{w}}^{\text {in }(\text { out })}(a, f)$ appartient à $H_{\bar{w}}^{\text {as }}$ d'où :

$$
\sum_{n=1}^{\infty} \oplus H_{\bar{w}}^{\text {in }}\left(f_{n}\right) \subseteq H_{\bar{w}}^{\text {as }} \sum_{n=1}^{\infty} \oplus H_{\bar{w}}^{\text {out }}\left(f_{n}\right) \subseteq H_{\bar{w}}^{\text {as }} .
$$

Il nous reste à prouver que l'inclusion peut être remplacée par une égalité. La démonstration de cette assertion est tout à fait identique à celle de la proposition 3 de l'article [5], c'est pourquoi nous l'omettrons.

Passons maintenant à l'étude de la matrice $S_{\bar{v}}$. Il est clair que

$$
S_{\bar{w}} \psi_{\bar{w}}(1, f)=\psi_{\bar{w}}(1, f) \quad S_{\bar{w}} H_{\bar{w}}^{\text {in }}(f)=H_{\bar{w}}^{\text {out }}(f)
$$

pour toute fonction $f \in L_{2}(\mu, s)$.

Il est naturel de s'intéresser aux sous-espaces de $H_{\bar{w}}^{\text {as }}$ stables à la fois pour $A_{\bar{w}}^{\text {in }}(x)$ et pour $S_{\bar{w}}$ (donc, et pour $A_{\bar{w}}^{\text {out }}(x)$ ). Un tel sous-espace étant trouvé, rien n'empêche de le prendre pour espace asymptotique du système (cela signifiant que les états observables appartiennent tous à ce sous-espace).

Soit donc un tel sous-espace. Il est stable pour $A_{\bar{w}}^{\text {in }}(x)$ par hypothèse, donc, en contenant un vide $\psi_{\bar{w}}(1, f)$ il contient tous le sous-espace $H_{\bar{w}}^{\text {in }}(f)$. Il en résulte que le problème peut être formulé de la façon suivante: $L$ étant un sous-espace de $L_{2}(\mu, s)$, quelle est la condition nécessaire et suffisante pour que le sous-espace

soit stable pour $S_{\bar{w}}$ ?

$$
H_{\bar{w}}^{\text {in }}(L) \equiv \bigcup_{f \in L} H_{\bar{w}}^{\text {in }}(f) \leqq H_{\bar{w}}^{\text {as }}
$$

Soient $a, b$ deux éléments de $A$. Désignons :

$$
S_{a b}(w) \equiv\left\langle\psi_{w}^{\text {in }}(a), \psi_{w}^{\text {out }}(b)\right\rangle .
$$

Il est évident que la fonction $S_{a b}(w)$ est bornée sur $s$. Nous la supposons en outre mesurable. 
Remarque. Dans les cas concrets lorsqu'on peut définir $S_{a b}(w)$ comme limite simple d'une suite des fonctions continues (voir le paragraphe suivant) la mesurabilité de $S_{a b}(w)$ résulte du théorème de EGorofF [4].

Proposition 2. Pour que le sous-espace $H_{\bar{w}}^{\text {in }}(L)$ soit stable pour $S_{\bar{w}} i l$ faut et il suffit que $L$ soit stable pour multiplication par toute fonction $S_{a b}(w)$ :

$$
f(w) \in L \Rightarrow S_{a b}(w) f(w) \in L \quad \forall a, b \in A .
$$

Démonstration. Notons: $L^{\prime} \equiv L_{2}(\mu, s) \ominus L$. Il est clair que $H_{\bar{w}}^{\text {as }}$ $=H_{\bar{w}}^{\text {in }}(L) \oplus H_{\bar{w}}^{\text {in }}\left(L^{\prime}\right)$ d'où on voit que $H_{\bar{w}}^{\text {in }}(L)$ est stable pour $S_{\bar{w}}$ si et seulement si l'égalité

$$
\left\langle\psi_{\bar{w}}^{\text {in }}(a, h), \psi_{\bar{w}}^{\text {out }}(b, f)\right\rangle=0
$$

est valide pour tous les $a, b \in A$ et toutes fonctions $f \in L, h \in L^{\prime}$. On a :

$$
\left\langle\psi_{\bar{w}}^{\mathrm{in}}(a, h), \psi_{\bar{w}}^{\mathrm{out}}(b, f)\right\rangle=\int_{s} S_{a b}(w) h^{*}(w) f(w) d \mu(w)
$$

d'où la proposition.

Corollaire. Pour qu'un sous-espace $H_{\bar{w}}^{\mathrm{in}}(f)$ soit stable pour $S_{\bar{w}}$ il faut et il suffit que la fonction $S_{a b}(w)$ soit constante sur le support de $f$ pour tous $a, b \in A$.

Il en résulte qu'en général aucun des sous-espaces $H_{\bar{w}}^{\text {in }}(f)$ n'est stable pour $S_{\bar{w}}$, ce qui signifie qu'il existe des amplitudes de transition non nulles entre des excitations au-dessus des vides différents.

Remarque. Du point de vue de l'interprétation considérée les deux fonctionelles:

$$
\bar{w}_{1}=\int_{s} w d \mu_{1}(w) \quad \bar{w}_{2}=\int_{s} w d \mu_{2}(w)
$$

décrivent la même théorie physique si les mesures $\mu_{1}$ et $\mu_{2}$ sont équivalentes. En effet, soit $f_{1 i} i=1,2, \ldots$ une base orthonormale dans $L_{2}\left(\mu_{1}, s\right)$. Alors l'ensemble des fonctions $f_{2 i}(w)=f_{1 i}(w) \varphi(w)$ où $\varphi=\left(\frac{d \mu_{1}}{d \mu_{2}}\right)^{1 / 2}$ constitue une base orthonormale dans $L_{2}\left(\mu_{2}, s\right)$. On a:

$$
\left\langle\psi_{\bar{w}_{1}}^{\text {in }}\left(a, f_{1 i}\right), S_{\bar{w}_{1}} \psi_{\bar{w}_{1}}^{\text {in }}\left(b, f_{1 k}\right)\right\rangle=\left\langle\psi_{\bar{w}_{2}}^{\text {in }}\left(a, f_{2 i}\right), S_{\bar{w}_{2}} \psi_{\bar{w}_{2}}^{\text {in }}\left(b, f_{2 k}\right)\right\rangle
$$

d'où on voit que ces deux théories se confondent si l'on identifie

$$
\psi_{\bar{w}_{\mathbf{2}}}^{\text {in }}\left(a, f_{2}\right) \quad \text { à } \quad \psi_{\bar{w}_{1}}^{\text {in }}\left(a, f_{1 i}\right) .
$$

\section{Rétablissement de la symétrie, dégénérescence du vide et formalisme du spurion}

Dans ce paragraphe nous étudions cette construction asymptotique du point de vue de la symétrie.

Soit $w$ une fonctionelle de la classe $Q_{0}$ non invariante par un groupe $T \subset T^{0}[5]$. Si $T$ est compact, on peut définir «la moyenne» :

$$
\bar{w}=\frac{1}{\mu(T)} \int_{\tau} w_{\tau} d \mu(\tau)
$$


où $w_{\tau} \in Q_{0}[5]$ est définie par l'équation:

$$
w_{\imath}(a)=w(\tau a) \quad w=w_{1}
$$

et $\mu$ est une mesure sur $T$ invariante à droite:

$$
d \mu\left(\tau \tau^{\prime}\right)=d \mu(\tau) \quad \forall \tau^{\prime} \in T .
$$

La fonctionelle $\bar{w}$ est $T$-invariante par construction. On peut vérifier (ce qui a été fait dans [5] pour un groupe de jauge) que la fonctionelle $\bar{w}$ appartient à la classe $Q$ pour tout groupe compact $T \subset T^{0}$.

Nous nous intéressons à la relation entre les théories physiques correspondantes aux fonctionelles $w$ et $\bar{w}$.

Pour parler concrètement considérons le cas où $T$ est un groupe de jauge. Soit donc $w \in Q_{0}$ une fonctionelle décrivant une représentation du champ complexe $A_{i}(x) i=1,2$ où $A_{1}(x) \equiv A(x), A_{2}(x) \equiv A^{+}(x)$. Supposons que cette fonctionelle n'est pas invariante par le groupe de jauge du champ $A_{i}(x)$ :

$$
A_{i}(x) \rightarrow A_{i}(x) \exp i \alpha \varepsilon_{i}, \quad \varepsilon_{1}=-1, \varepsilon_{2}=1 .
$$

Soit $G \equiv\left\{g(\alpha): \alpha \in R^{1}\right\}$ le groupe des *-automorphismes de l'algèbre $A$ représentant ce groupe de jauge:

$$
(g(\alpha) a)_{i_{1} \ldots i_{n}}\left(x_{1} \ldots x_{n}\right)=a_{i_{1} \ldots i_{n}}\left(x_{1} \ldots x_{n}\right) \exp i \alpha \sum_{k=1}^{n} \varepsilon_{i_{k}}, \quad i_{\alpha}=1,2 .
$$

Notons:

$$
w \equiv w_{0} \quad w_{\alpha}(a) \equiv w(g(\alpha) a) .
$$

Les fonctions de Wightman $w_{i_{1} \ldots i_{n}}^{(\alpha)}\left(x_{1} \ldots x_{n}\right)$ dépendent de $\alpha$ par l'intermédiaire du facteur $\exp i \alpha N\left(i_{1} \ldots i_{n}\right)$ où $N\left(i_{1} \ldots i_{n}\right)$ est le nombre de champs $A_{2}$ moins celui de champs $A_{1}$ contenus dans la fonction de Wightman considérée. La non invariance de $w$ signifie qu'il existe des fonctions $w_{i_{1}}^{(0)} \ldots i_{n}\left(x_{1} \ldots x_{n}\right)$ non nulles telles que $N\left(i_{1} \ldots i_{n}\right) \neq 0$. Il a été montré [5] que l'ensemble des fonctions

$$
\left\{\exp i \propto N\left(i_{1} \ldots i_{n}\right): w_{i_{1} \ldots i_{n}}^{(0)} \neq 0\right\}
$$

constitue un groupe, autrement dit, il est de la forme

$$
\{\exp i \alpha m n: n=0, \pm 1, \pm 2, \ldots\}
$$

où $m$ est un entier fixe. Les fonctions $w_{\alpha}(a)$ ( $a \in A$ est fixé) appartiennent à l'espace $C_{0}(2 \pi / m)$ des fonctions continues périodiques de période $2 \pi / m$ et constituent un ensemble dense dans cet espace en vertu du lemme 3 de l'article [5].

Pour rétablir la symétrie $G$ il faut passer à la fonctionelle

$$
\bar{w}=\frac{m}{2 \pi} \int_{0}^{2 \pi / m} w_{\alpha} d \alpha .
$$


Ces deux cas sont physiquement tout à fait différents. Dans le premier cas les particules asymptotiques portent une $G$ charge définie, mais En comparant avec la forme canonique des fonctionelles de la classe $Q$ on voit que $s=\left\{w_{\alpha}: \alpha \in[0,2 \pi / m)\right\}([0,2 \pi / m)$ désigne l'intervalle demiouvert) et $d \mu\left(w_{\alpha}\right)=\frac{m}{2 \pi} d \alpha$. Dorénavant nous désignerons l'espace $L_{2}(\mu, s)$ plus simplement par $L_{2}$.

Nous désirons comparer les théories physiques correspondantes aux fonctionelles $w$ et $\bar{w}$. Commençons par la fonctionelle $w$ et supposons pour simplifier que cette fonctionelle engendre un seul champ complexe asymptotique de la masse $m$.

Ecrivons les premières fonctions de Wightman de cette fonctionelle:

$$
\begin{aligned}
w_{i}(x) & =\left\langle\psi_{w}(1), A_{i}(x) \psi_{w}(1)\right\rangle \equiv h_{i}, \\
w_{i k}\left(x_{1} x_{2}\right) & =\int d p e^{i p\left(x_{1}-x_{2}\right)} \theta\left(p_{0}\right) \varrho_{i k}\left(p^{2}\right) .
\end{aligned}
$$

La première démarche du procédé de Haag-Ruelle est la construction du champ auxiliaire $B_{i}(x)$ (champ d'interpolation). Pour faciliter ce problème supposons que:

$$
\varrho_{i k}\left(p^{2}\right)=d_{i k} \delta\left(p^{2}-m^{2}\right)+\sigma_{i k}\left(p^{2}\right)
$$

où $\sigma_{i k}\left(p^{2}\right)$ est nulle dans un voisinage du point $p^{2}=m^{2}$.

Alors on peut prendre

$$
\widetilde{B}_{i}(p)=\lambda\left(p^{2}\right) \sum_{k=1}^{2} a_{i k} \widetilde{A}_{k}(p)+c_{i}
$$

où $\widetilde{B}_{i}(p), \widetilde{A}_{i}(p)$ sont les transformées de Fourier des champs $B_{i}(x), A_{i}(x)$ respectivement, $\lambda\left(p^{2}\right)$ est une fonction de l'espace de Schwartz à support compact contenu dans un voisinage du point $p^{2}=m^{2}$ de sorte que le produit $\lambda\left(p^{2}\right) \sigma_{i k}\left(p^{2}\right)$ soit nul, $a_{i k}, c_{i}$ - des nombres complexes qu'on doit choisir de façon que les premières fonctions de Wightman du champ $B_{i}(x)$ soient identiques à celles du champ complexe libre de la masse $m$, de façon précise:

$$
\begin{aligned}
\left\langle\psi_{w}(1), B_{i}(x) \psi_{w}(1)\right\rangle & =0 \\
\left\langle\psi_{w}(1), B_{i}\left(x_{1}\right) B_{k}\left(x_{2}\right) \psi_{w}(1)\right\rangle & =\frac{1}{(2 \pi)^{3}} \int d p e^{i p\left(x_{1}-x_{2}\right)} \theta\left(p_{0}\right) \delta\left(p^{2}-m^{2}\right) b_{i k}
\end{aligned}
$$

où $b_{11}=b_{22}=0, b_{12}=b_{21}=1$.

Si la fonctionelle $w$ était $G$ invariante, on aurait: $h_{i}=0 \varrho_{11}=\varrho_{22}=0$ et par suite $c_{i}=0, a_{12}=a_{21}=0 . w$ n'étant pas supposée $G$ invariante, on doit distinguer deux cas:

1. $w$ n'est pas $G$ invariante, mais la partie qui intervient dans la construction de $B_{i}(x)$ est $G$ invariante: $h_{i}=0, d_{1 \mathfrak{1}}=d_{22}=0$. Dans ce cas $c_{i}=0, a_{12}=a_{21}=0$.

2. Même cette partie n'est pas $G$ invariante. 
l'interaction ne conserve pas cette charge. Cette situation est comparable à la non-conservation de l'étrangeté dans la théorie de l'interaction faible.

Dans le deuxième cas on ne peut plus attribuer de $G$ charge définie aux particules asymptotiques ce qui se passe par exemple avec la charge éléctrique dans la théorie de la superconductivité.

Formulons le passage de $A_{i}(x)$ à $B_{i}(x)$ d'une facon compacte. Soit $r_{w}(\alpha) \equiv \sum_{n} \sum_{i_{1} \ldots i_{n}=1}^{2} \int \cdots \int d x_{1} \ldots d x_{n} a_{i_{1} \ldots i_{n}}\left(x_{1} \ldots x_{n}\right) A_{i_{1}}\left(x_{1}\right) \ldots A_{i_{n}}\left(x_{n}\right)$.

Désignons par $\tilde{r}_{w}(a)$ la même expression où $A_{i}(x)$ est remplacé par $B_{i}(x)$. Alors l'équation $\tilde{r}_{w}(a)=r_{w}(h a)$ définit un *-homomorphisme de l'algèbre $A$ sur elle-même. Cet homomorphisme est permutable avec $G$ dans le premier cas et non permutable dans le deuxième cas.

Le champ $B_{i}(x)$ étant construit, définissons:

$$
\psi_{w}^{\text {in (out) }}(a)=\lim _{t \rightarrow-(+) \infty} \psi_{w}\left(h g_{t} a\right)
$$

où $g_{t}$ est le *-automorphisme de $A$ :

$$
\begin{aligned}
\widetilde{\left(g_{t} a\right)_{i_{1} \ldots i_{n}}}\left(p_{1} \ldots p_{n}\right) & =\widetilde{a}_{i_{1} \ldots i_{n}}\left(p_{1} \ldots p_{n}\right) \exp i t \sum_{k=1}^{n}\left(p_{k 0}-\bar{\varepsilon}\left(p_{k 0}\right) p_{k 0}^{m}\right) \\
p_{k} & \equiv\left(p_{k 0}, \boldsymbol{p}_{k}\right) \quad p_{k 0}^{m} \equiv\left(m^{2}+\boldsymbol{p}_{k}^{2}\right)^{1 / 2}
\end{aligned}
$$

et $\bar{\varepsilon}\left(p_{0}\right)$ est une fonction indéfiniment différentiable caractérisée par les propriétés suivantes: 1. $\bar{\varepsilon}\left(-p_{0}\right)=-\bar{\varepsilon}\left(p_{0}\right), 2$. elle coincide avec $\varepsilon\left(p_{0}\right)$ $\equiv \theta\left(p_{0}\right)-\theta\left(-p_{0}\right)$ partout sauf un voisinage suffisamment petit du point $p_{0}=0$.

Nous supposons que la limite en question existe dans la norme de l'espace $H_{w}$ pour tout $a \in A$ ou au moins pour un ensemble topologiquement dense dans $A$ et que $\psi_{w}^{\text {in (out) }}(a)$ sont des états d'un champ libre en ce sens que les fonctionelles

$$
w^{\text {in } \text { (out) }}(a) \equiv\left\langle\psi_{w}(1), \psi_{w}^{\text {in (out) }}(a)\right\rangle
$$

sont égales toutes deux à la fonctionelle de Wightman du champ complexe libre de la masse $m$. Si $w \in Q_{0}$ est locale, ce n'est plus une supposition mais une assertion prouvée par D. Ruelle [2].

Notons:

$$
w^{\text {as }}(a) \equiv w^{\text {in }}(a)=w^{\text {out }}(a) .
$$

L'application $S_{w} \psi_{w}^{\text {in }}(a)=\psi_{w}^{\text {out }}(a)$ est l'isométrie de sous-espace $H_{w}^{\text {in }}$ [la fermeture de l'ensemble $\left.\psi_{w}^{\text {in }}(a): a \in A\right]$ sur $H_{w}^{\text {out }}$ [la fermeture de l'ensemble $\left.\psi_{w}^{\text {out }}(a): a \in A\right]$. Pour que $S_{w}$ soit unitaire il faut et il suffit que les sousespaces $H_{w}^{\text {in }}$ et $H_{w}^{\text {out }}$ soient identiques [2]. Nous le supposons et désignons $H_{w}^{\text {in }}=H_{w}^{\text {out }}$ par $H_{w}^{\text {as }}$. 
La fonctionelle $w^{\text {as }}$ est par construction invariante par les transformations de jauge du champ $A_{i}^{\text {in (out) }}(x): w^{\text {as }}(g(\alpha) a)=w^{\text {as }}(a)$. Nous nous intéressons aussi aux propriétés de symétrie par rapport aux transformations de jauge du champ $A_{i}(x)$.

Si l'on fait subir au champ $A_{i}(x)$ une transformation de jauge $A_{i}(x) \rightarrow A_{i}(x) \exp i \alpha \varepsilon_{i}$, on voit que

$$
\begin{gathered}
\tilde{r}_{w}(a)=r_{w}(h a) \rightarrow r_{w}(g(\alpha) h a) \\
\psi_{w}\left(h g_{t} a\right) \rightarrow \psi_{w}\left(g(\alpha) h g_{t} a\right)
\end{gathered}
$$

Il est évident que dans les deux cas (interaction faible et super-conductivité) l'équation $g(\alpha) h=h g_{h}(\alpha)$ définit un *automorphisme $g_{h}(\alpha)$ de l'algèbre $A$. Dans le premier cas $g_{h}(\alpha)$ est identique à $g(\alpha)$, dans le deuxième cas ce sont des automorphismes différents. On voit donc qu'une transformation de jauge du champ $A_{i}(x)$ engendre la transformation $\psi_{w}^{\text {in (out) }}(a) \rightarrow \psi_{w}^{\text {in (out) }}\left(g_{h}(\alpha) a\right)$ des états asymptotiques. Il est clair que la fonctionelle asymptotique est invariante par rapport à ces transformations dans le premier cas et qe'elle est non-invariante dans le deuxième cas. Par conséquent c'est seulement dans le premier cas que nous pouvons introduire la notion de la charge des particules asymptotiques associée au groupe de jauge du champ $A_{i}(x)$.

Etant donné $g_{h}(\alpha)=g(\alpha)$, construisons deux représentations unitaires du groupe G:

$$
\psi_{w}^{\text {in (out) }}(g(\alpha) a)=U_{w}^{\text {in (out) }}(\alpha) \psi_{w}^{\text {in (out) }}(a) .
$$

En vertu de la définition:

$$
U_{w}^{\text {in (out) }}(\alpha) A_{i w}^{\text {in (out) }}(x) U_{w}^{\text {in (out) }}(\alpha)^{-1}=A_{i w}^{\text {in (out) }}(x) \exp i \alpha \varepsilon_{i}
$$

d'où on voit que les charges correspondantes

$$
U_{w}^{\text {in (out) }}(\alpha)=\exp i \alpha Q_{w}^{\text {in (out) }}
$$

peuvent être représentées comme l'opérateur du nombre de particules moins celui du nombre des antiparticules in (out) respectivement si $A_{1 w}^{\text {in (out) }}(x)$ contient comme d'habitude l'opérateur de destruction de la particule et celui de création de l'antiparticule in (out) respectivement et $A_{2 w}^{\text {incout }}(x)$ - les opérateurs conjugués.

Les deux représentations sont unitairement équivalentes:

$$
U_{w}^{\text {out }}(\alpha)=S_{w} U_{w}^{\text {in }}(\alpha) S_{w}^{-1}
$$

et comme $w$ n'est pas $G$ invariante

$$
U_{w}^{\text {out }}(\alpha) \neq U_{w}^{\text {in }}(\alpha)
$$

ce qui signifie que $U_{w}^{\text {in }}(\alpha)$ n'est pas permutable avec la matrice $S_{w}$ [de même pour $U_{w}^{\text {out }}(\alpha)$ ]. 
Remarque. Il peut arriver que la matrice $S_{w}$ conserve la charge (autrement dit, $U_{w}^{\text {in }}(\alpha)=U_{w}^{\text {out }}(\alpha)$ ) bien que la fonctionelle $w$ ne soit pas $G$ invariante. Nous supposons que ce n'est pas le cas.

Soit $a_{n, \bar{n}}$ un élément de $A$ tel que $\psi_{w}^{\text {in }}\left(a_{n, \bar{n}}\right)$ contienne $n$ particules et $\bar{n}$ antiparticules. Evidemment

$$
Q_{w}^{\text {in }} \psi_{w}^{\text {in }}\left(a_{n, \bar{n}}\right)=(n-\bar{n}) \psi_{w}^{\text {in }}\left(a_{n, \bar{n}}\right) .
$$

L'inégalité $Q_{w}^{\text {in }} \neq=Q_{w}^{\text {out }}$ signifie qu'il existe deux états asymptotiques $\psi_{w}^{\text {in }}\left(a_{n, \bar{n}}\right), \psi_{w}^{\text {in }}\left(b_{n^{\prime}, \bar{n}^{\prime}}\right)$ de charges différentes $\left(n-\bar{n} \neq n^{\prime}-\bar{n}^{\prime}\right)$ tels que l'amplitude de transition

ne soit pas nulle.

$$
\left\langle\psi_{w}^{\text {in }}\left(a_{n, \bar{n}}\right), S_{w} \psi_{w}^{\text {in }}\left(b_{n^{\prime}, \bar{n}^{\prime}}\right)\right\rangle
$$

Dans le cas considéré cette amplitude de transition est engendrée par la fonction de Wightman contenant $n^{\prime}+\bar{n}$ de champs $A_{2}$ et $n+\bar{n}^{\prime}$ champs $A_{1}$. En vertu de l'hypothèse sur $w$, pour que cette amplitude ne soit pas nulle il faut que le nombre $n^{\prime}+\bar{n}-n-\bar{n}^{\prime}$ soit multiple de $m$, autrement dit, le changement de la charge satisfait à la regle de selection suivante: $\Delta Q$ est multiple de $m$.

Passons maintenant à la théorie correspondante à «la moyenne»:

$$
\bar{w}=\frac{m}{2 \pi} \int_{0}^{2 \pi / m} w_{\alpha} d \alpha
$$

construite à partir de $w \equiv w_{0}$. Dans cet article nous nous bornons à l'analyse du premier cas seulement (interaction faible). Le résultat sera le suivant: si l'on interprete la théorie $\bar{w}$ de la façon décrite dans les paragraphes précédents cette théorie est physiquement identique à la théorie initiale $w$. Tout ce qui suit est la démonstration de cette assertion.

Prouvons tout d'abord que la fonctionelle $\bar{w}$ satisfait aux conditions 1 - 3 du paragraphe précédent ce qui permet d'appliquer les propositions 1,2 .

Il est évident que:

1. on peut utiliser le même homomorphisme $h$ pour toute fonctionelle $w_{\alpha}: \alpha \in e \equiv[0,2 \pi / m)$,

2. si la construction de Haag-Ruelle marche bien pour $w \equiv w_{0}$, (ce qui est supposé), elle marche bien pour toute $w_{\alpha}: \alpha \in e$ et par suite on peut définir correctement les champs asymptotiques $A_{w_{\alpha}}^{\text {in (out) }}(x)$, la fonctionelle asymptotique $w_{\alpha}^{\text {as }}$, la matrice $S_{w_{\alpha}}$,

3. les fonctionelles asymptotiques sont les mêmes:

$$
w_{\alpha}^{\text {as }}=w_{\alpha^{\prime}}^{\text {as }} \quad \forall \alpha, \alpha^{\prime} \in e .
$$

Seule l'unitarité de la matrice $S_{w_{\alpha}}$ n'est pas évidente.

Lemme. Si $S_{w_{0}}$ est unitaire, il en est de même de $S_{w_{\alpha}} \forall \alpha \in e$. 
Démonstration. Nous devons donc montrer que l'égalité $H_{w_{0}}^{\text {in }}=H_{w_{0}}^{\text {out }}$ entraine $H_{w_{\alpha}}^{\text {in }}=H_{w_{\alpha}}^{\text {out }}$ pour tout $\alpha \in e$.

Soit $a_{n}: n=1,2, \ldots$ une suite d'éléments de $A$ telle que l'ensemble $\psi_{w_{0}}^{\text {in }}\left(a_{n}\right): n=1,2, \ldots$ soit une base orthonormale dans $H_{w_{0}}^{\text {in }}$ (une telle suite évidemment existe). Alors l'ensemble $\psi_{w_{\alpha}}^{\text {in }}\left(a_{n}\right): n=1,2, \ldots$ est une base orthonormale dans $H_{w_{\alpha}}^{\text {in }}$ en vertu de l'équivalence unitaire entre $A_{w_{0}}^{\text {in }}(x)$ et $A_{w_{\alpha}}^{\text {in }}(x)$. Prouvons que $H_{w_{\alpha}}^{\text {in }} \supseteq H_{w_{\alpha}}^{\text {out }}$. Pour faire cela il suffit de vérifier que l'égalité:

$$
\left\langle\psi_{w_{\alpha}}^{\text {out }}(a), \psi_{w_{\alpha}}^{\text {out }}(b)\right\rangle=\sum_{n=1}^{\infty}\left\langle\psi_{w_{\alpha}}^{\text {out }}(a), \psi_{w_{\alpha}}^{\text {in }}\left(a_{n}\right)\right\rangle\left\langle\psi_{w_{\alpha}}^{\text {in }}\left(a_{n}\right), \psi_{w_{\alpha}}^{\text {out }}(b)\right\rangle
$$

est valide pour les $a, b \in A$. Comme cette égalité est vraie pour $w_{0}$ par hypothèse il est naturel de chercher une relation entre des amplitudes de transition correspondantes. On a:

$$
\begin{aligned}
\left\langle\psi_{v v_{\alpha}}^{\text {in }}(a),\right. & \left.\psi_{2 l \alpha_{\alpha}}^{\text {out }}(b)\right\rangle=\lim _{\substack{t_{1} \rightarrow-\infty \\
t_{2} \rightarrow+\infty}}\left\langle\psi_{v v_{\alpha}}\left(h g_{t_{1}} a\right), \psi_{3 l_{\alpha}}\left(h g_{t_{2}} b\right)\right\rangle \\
& =\lim _{\substack{t_{1} \rightarrow-\infty \\
t_{2} \rightarrow+\infty}} w_{\alpha}\left(\left(h g_{t_{1}} a\right)^{+} \cdot h g_{t_{2}} b\right)=\lim _{\substack{t_{1} \rightarrow-\infty \\
t_{2} \rightarrow+\infty}} w_{0}\left(g(\alpha)\left[\left(h g_{t_{1}} a\right)^{+} \cdot h g_{t_{2}} b\right]\right) \\
& =\lim _{\substack{t_{1} \rightarrow-\infty \\
t_{2} \rightarrow+\infty}} w_{0}\left(\left(h g_{t_{1}} g(\alpha) a\right)^{+} \cdot h g_{t_{2}} g(\alpha) b\right) \\
& =\left\langle\psi_{w_{0}}^{\text {in }}(g(\alpha) a), \psi_{w_{0}}^{\text {out }}(g(\alpha) b)\right\rangle .
\end{aligned}
$$

Il vient:

$$
\begin{aligned}
& \left\langle\psi_{w_{\alpha}}^{\text {out }}(a), \psi_{w_{\alpha}}^{\text {out }}(b)\right\rangle \\
& \quad=\sum_{n=1}^{\infty}\left\langle\psi_{w_{0}}^{\text {out }}(g(\alpha) a), \psi_{w_{0}}^{\text {in }}\left(g(\alpha) a_{n}\right)\right\rangle\left\langle\psi_{w_{0}}^{\text {in }}\left(g(\alpha) a_{n}\right), \psi_{w_{0}}^{\text {out }}(g(\alpha) b)\right\rangle .
\end{aligned}
$$

Puisque $\psi_{w_{0}}^{\text {in }}\left(g(\alpha) a_{n}\right)=U_{w_{0}}^{\text {in }}(\alpha) \psi_{w_{0}}^{\text {in }}\left(a_{n}\right)$, l'ensemble $\psi_{w_{0}}^{\text {in }}\left(g(\alpha) a_{n}\right): n=1,2, \ldots$ est aussi une base orthonormale dans $H_{w_{0}}^{\text {in }}$ et par suite le membre droit de l'équation ci-dessus est égale à

$$
\left\langle\psi_{w_{0}}^{\text {out }}(g(\alpha) a), \psi_{w_{0}}^{\text {out }}(g(\alpha) b)\right\rangle=\left\langle\psi_{w_{0}}^{\text {out }}(a), \psi_{w_{0}}^{\text {out }}(b)\right\rangle=\left\langle\psi_{w_{\alpha}}^{\text {out }}(a), \psi_{w_{\alpha}}^{\text {out }}(b)\right\rangle
$$

d' où l'assertion.

En permutant (in) et (out) dans le raisonnement on peut démontrer l'inclusion inverse ce qui acheve la démonstration du lemme.

En appliquant la construction du paragraphe précédent introduisons:

$$
\begin{aligned}
H_{\bar{w}}^{\mathrm{as}} & =\frac{m}{2 \pi} \int_{e} \oplus H_{w_{\alpha}}^{\mathrm{as}} d \alpha \quad A_{\bar{w}}^{\mathrm{in}(\text { out })}(x)=\int_{e} \oplus A_{w_{\alpha}}^{\mathrm{in}(\text { out })}(x), \\
S_{\bar{w}} & =\int_{e} \oplus S_{w_{\alpha}} .
\end{aligned}
$$

Le sous-espace de vide $H_{\bar{w}}^{0} \subset H_{\bar{w}}^{\text {as }}$ est isomorphe à $L_{2}$. L'ensemble des fonctions $f_{n}(\alpha)=\exp i \alpha m n: n=0, \pm 1, \pm 2, \ldots$ étant une base orthonormale de $L_{2}$, l'ensemble des vides $\psi_{\bar{w}}\left(1, f_{n}\right): n=0, \pm 1, \ldots$ constitue 8 Commun. math. Phys., Vol. 13 
une base orthonormale dans $H_{\bar{w}}^{0}$. En vertu de la proposition 1

$$
H_{\bar{w}}^{\text {as }}=\sum_{n=-\infty}^{\infty} \oplus H_{\bar{w}}^{\text {in }}\left(f_{n}\right)=\sum_{n=-\infty}^{\infty} \oplus H_{\bar{w}}^{\text {out }}\left(f_{n}\right) .
$$

Considérons une fonction

$$
S_{a b}(\alpha) \equiv\left\langle\psi_{u u_{\alpha}}^{\text {in }}(a), \psi_{w_{\alpha}}^{\text {out }}(b)\right\rangle .
$$

Il est clair qu'on peut choisir $a, b \in A$ de sorte que cette fonction ne soit pas constante sur $e$. En effet, d'après la démonstration ci-dessus:

$$
\begin{aligned}
S_{a b}(\alpha) & =\left\langle\psi_{w_{0}}^{\text {in }}(g(\alpha) a), S_{w_{0}} \psi_{w_{0}}^{\text {in }}(g(\alpha) b)\right\rangle \\
& =\left\langle U_{w_{0}}^{\text {in }}(\alpha) \psi_{w_{0}}^{\text {in }}(a), S_{w_{0}} U_{w_{0}}^{\text {in }}(\alpha) \psi_{w_{0}}^{\text {in }}(b)\right\rangle .
\end{aligned}
$$

Comme $U_{w_{0}}^{\text {in }}(\alpha)$ ne commute pas avec $S_{w_{0}}$ par hypothèse, il existe $a, b \in A$ tels que

$$
\left\langle U_{w_{0}}^{\text {in }}(\alpha) \psi_{w_{0}}^{\text {in }}(a), S_{w_{3}} U_{w_{0}}^{\text {in }}(\alpha) \psi_{w_{0}}^{\text {in }}(b)\right\rangle \neq\left\langle\psi_{w_{0}}^{\text {in }}(a), S_{w_{0}} \psi_{w_{0}}^{\text {in }}(b)\right\rangle \equiv S_{a b}(0)
$$

d'où l'assertion.

En appliquant le corollaire de la proposition 2 on voit qu'aucun des sous-espaces $H_{\bar{w}}^{\text {in }}\left(f_{n}\right)$ n'est stable pour $S_{\bar{w}}$.

Passons à la symétrie. On peut construire les trois représentations du groupe $G$. Tout d'abord on a

$$
U_{\bar{w}}^{\text {in }(\text { out })}(\alpha)=\int_{e} \oplus U_{w_{\alpha^{\prime}}}^{\text {in }(\text { out })}(\alpha)
$$

( $\alpha^{\prime}$ est une variable muette) où $U_{x^{\prime} \alpha^{\prime}}^{\text {infout) }}(\alpha)$ sont les opérateurs unitaires dans $H_{w_{\alpha^{\prime}}}^{\text {as }}$, définis par l'équation:

$$
U_{w \alpha^{\prime}}^{\text {in (out) }}(\alpha) \psi_{w \alpha^{\prime}}^{\text {in (out) }}(\alpha)=\psi_{w^{\prime} \alpha^{\prime}}^{\text {in (out) }}(g(\alpha) a) .
$$

Les charges correspondantes:

$$
U_{\bar{w}}^{\text {in (out) }}(\alpha)=\exp i \alpha Q_{\bar{w}}^{\text {in (out) }} \quad Q_{\bar{w}}^{\text {in (out) }}=\int_{e} \oplus Q_{w_{\alpha^{\prime}}}^{\text {in (out) }}
$$

seront appelées charges des particules car pour tout état asymptotique contenant $n$ particules et $\bar{n}$ antiparticules excitées au-dessus de n'importe quel vide cette charge est égale à $n-\bar{n}$.

Définissons deux opérateurs:

$$
Q_{\bar{w}, \text { vide }}^{\text {in (out) }} \equiv \sum_{n=-\infty}^{\infty} m n P_{n}^{\text {in (out) }}
$$

où $P_{n}^{\text {in (out) }}$ sont les projecteurs sur $H_{\bar{w}}^{\text {in (out) }}\left(f_{n}\right)$ respectivement. Ces opérateurs seront appelées charges asymptotiques du vide. Par définition la charge in(out) du vide de tout vecteur de $H_{\bar{w}}^{\text {in }(\text { out })}\left(f_{n}\right)$ est égale à $m n$.

Soient $U_{\bar{w}, \text { vide }}^{\text {in }(\text { out) }}(\alpha)=\exp i \alpha Q_{\bar{w} \text {, vide }}^{\text {in (out) }}$ les représentations unitaires du groupe $G$ engendrées par les charges du vide. On peut vérifier que pour tout vecteur $\psi_{\bar{w}}^{\text {in }}$ (out) $(a, f)$

$$
U_{\bar{w}, \text { vide }}^{\text {in }(\text { out })}(\alpha) \psi_{\bar{w}}^{\text {in }(\text { out })}(a, f)=\psi_{\bar{w}}^{\text {in }(\text { out })}\left(a, f_{\alpha}\right)
$$


où $f_{\alpha}\left(\alpha^{\prime}\right)=f\left(\alpha+\alpha^{\prime}\right)$, autrement dit, la restriction de la représentation $U_{\bar{u}}^{\text {in } \text {, vide }}(\alpha)$ au sous-espace de vide constitue la représentation regulière du groupe $G$.

Il est évident que la matrice $S_{\vec{w}}$ ne conserve ni la charge des particules, ni celle du vide.

L'invariance de la fonctionelle $\bar{w}$ permet de construire encore une représentation du groupe $G$. Les opérateurs unitaires de cette représentation sont définis par l'équation [5]:

$$
\left[U_{\bar{w}}(\alpha) x\right]_{w_{\alpha^{\prime}}}=V\left(w_{\alpha^{\prime}}, w_{\alpha+\alpha^{\prime}}\right)[x]_{w_{\alpha+\alpha^{\prime}}}
$$

où $x$ - un vecteur de $H_{\bar{w}},[x]_{w_{\alpha}}$ - sa composante dans $H_{w^{\prime} \alpha}, V\left(w_{\alpha^{\prime}}, w_{\alpha+\alpha^{\prime}}\right)$ - l'isométrie de $H_{w_{\alpha+\alpha^{\prime}}}$ sur $H_{w_{\alpha^{\prime}}}$ :

$$
V\left(w_{\alpha^{\prime}}, w_{\alpha+\alpha^{\prime}}\right) \psi_{w_{\alpha+\alpha^{\prime}}}(a)=\psi_{w_{\alpha^{\prime}}}(g(\alpha) a) .
$$

Désignons par $Q_{\bar{w}}$ la charge correspondante et démontrons

Proposition 3. La charge $Q_{\bar{w}}$ est la somme de la charge des particules et de celle du vide:

$$
Q_{\bar{w}}=Q_{\bar{w}}^{\mathrm{in}}+Q_{\bar{w}, \text { vide }}^{\text {in }}=Q_{\bar{w}}^{\text {out }}+Q_{\bar{w}, \text { vide }}^{\text {out }} .
$$

La charge $Q_{\bar{w}}$ se conserve dans l'interaction:

$$
S_{\bar{w}} Q_{\bar{w}}=Q_{\bar{w}} S_{\bar{w}}
$$

Démonstration. On peut facilement vérifier que les charges $Q_{\bar{v}}^{\text {in }}$ et $Q_{\bar{w} \text {, vide }}^{\text {in }}$ sont permutables. Prouvons que

$$
U_{\bar{w}}(\alpha)=U_{\bar{w}}^{\mathrm{in}}(\alpha) U_{\bar{w}, \text { vide }}^{\text {in }}(\alpha)
$$

sur $H_{\bar{w}}^{\text {as }}$. Pour $a \in A, f \in L_{2}$ fixés écrivons:

$$
\begin{aligned}
{\left[U_{\bar{w}}(\alpha) \psi_{\bar{w}}^{\mathrm{in}}(\alpha, f)\right]_{w_{\alpha^{\prime}}} } & =V\left(w_{\alpha^{\prime}}, w_{\alpha+\alpha^{\prime}}\right)\left[\psi_{\bar{w}}^{\mathrm{in}}(\alpha, f)\right]_{w_{\alpha+\alpha^{\prime}}} \\
& =f\left(\alpha+\alpha^{\prime}\right) V\left(w_{\alpha^{\prime}}, w_{\alpha+\alpha^{\prime}}\right) \psi_{w_{\alpha+\alpha^{\prime}}}^{\mathrm{in}}(a) \\
& =f\left(\alpha+\alpha^{\prime}\right) V\left(w_{\alpha^{\prime}}, w_{\alpha+\alpha^{\prime}}\right) \lim _{t \rightarrow-\infty} \psi_{w_{\alpha+\alpha^{\prime}}}\left(h g_{t} a\right) \\
& =f\left(\alpha+\alpha^{\prime}\right) \lim _{t \rightarrow-\infty} V\left(w_{\alpha^{\prime}}, w_{\alpha+\alpha^{\prime}}\right) \psi_{w_{\alpha+\alpha^{\prime}}}\left(h g_{t} a\right) \\
& =f\left(\alpha+\alpha^{\prime}\right) \lim _{t \rightarrow-\infty} \psi_{w_{\alpha^{\prime}}}\left(h g_{t} g(\alpha) a\right) \\
& =f\left(\alpha+\alpha^{\prime}\right) \psi_{w_{\alpha^{\prime}}}^{\text {in }}(g(\alpha) a)=\left[\psi_{\bar{w}}^{\text {in }}\left(g(\alpha) a, f_{\alpha}\right)\right]_{w_{\alpha^{\prime}}}
\end{aligned}
$$

d'où

$$
U_{\bar{w}}(\alpha) \psi_{\bar{w}}^{\mathrm{in}}(a, f)=\psi_{\bar{w}}^{\mathrm{in}}\left(g(\alpha) a, f_{\alpha}\right)=U_{\bar{u}^{\prime}}^{\mathrm{in}}(\alpha) U_{\bar{w}, \text { vide }}^{\mathrm{in}}(\alpha) \psi_{\bar{w}}^{\mathrm{in}}(a, f)
$$

En vertu de la proposition 1 l'ensemble $\psi_{\bar{w}}^{\text {in }}(a, f): a \in A, f \in L_{2}$ est dense dans $H_{\bar{w}}^{\text {as }}$, d'où l'assertion. donc

La démonstration est évidemment valide et pour $U_{\bar{w}}^{\text {out }}(\alpha), U_{\bar{w} \text {, vide }}^{\text {out }}(\alpha)$

$$
Q_{\bar{w}}=Q_{\bar{w}}^{\text {in }}+Q_{\bar{w}, \text { vide }}^{\text {in }}=Q_{\bar{w}}^{\text {out }}+Q_{\bar{w}, \text { vide }}^{\text {out }}
$$

et par conséquent $Q_{\bar{w}}=S_{\bar{w}} Q_{\bar{w}} S_{\bar{w}}^{-1}$ d'où la proposition. 
La symétrie de la fonctionelle $\bar{w}$ correspond donc à la conservation de la charge totale du système: les particules plus le vide. Puisqu'un observateur ne voit que ce qui se passe avec des particules pour lui il n'y a pas de différence entre les théories $w$ et $\bar{w}$ : toutes deux prédisent la non conservation de la charge des particules. La différence est seulement dans l'interprétation: dans le premier cas $(w)$ cette charge ne se conserve pas tout simplement, dans le deuxième $(\bar{w})$ - elle ne se conserve pas parce qu'elle passe des particules au vide.

Calculons maintenant des amplitudes de transition dans la théorie $\bar{w}$.

Soit $\psi_{\bar{w}}^{\text {in }}\left(a_{n, \bar{n}} ; f_{k}\right)$ un état asymptotique contenant $n$ particules, $\bar{n}$ antiparticules excitées au-dessus du vide de charge $m k$. On a :

$$
\begin{aligned}
& \left\langle\psi_{\bar{w}}^{\text {in }}\left(a_{n, \bar{n}} ; f_{k}\right), S_{\bar{w}} \psi_{\bar{w}}^{\text {in }}\left(b_{n^{\prime}, \bar{n}^{\prime}} ; f_{k^{\prime}}\right)\right\rangle \\
& =\frac{m}{2 \pi} \int_{0}^{2 \pi / m} d \alpha f_{k}^{*}(\alpha) f_{k^{\prime}}(\alpha)\left\langle\psi_{w_{\alpha}}^{\text {in }}\left(a_{n, \bar{n}}\right), S_{w_{\alpha}} \psi_{w_{\alpha}}^{\text {in }}\left(b_{n^{\prime}, \bar{n}^{\prime}}\right)\right\rangle \\
& =\frac{m}{2 \pi} \int_{0}^{2 \pi / m} d \alpha f_{k}^{*}(\alpha) f_{k^{\prime}}(\alpha)\left\langle\psi_{w_{0}}^{\text {in }}\left(g(\alpha) a_{n, \bar{n}}\right), S_{w_{0}} \psi_{w_{0}}^{\text {in }}\left(g(\alpha) b_{n^{\prime}, \bar{n}^{\prime}}\right)\right\rangle \\
& =\left\langle\psi_{w_{0}}^{\text {in }}\left(a_{n, \bar{n}}\right), S_{w_{0}} \psi_{w_{0}}^{\text {in }}\left(b_{n^{\prime}, \bar{n}^{\prime}}\right)\right\rangle \cdot \frac{m}{2 \pi} \int_{0}^{2 \pi / m} d \alpha \exp i \alpha \\
& \cdot\left(k^{\prime} m-k m+n^{\prime}-\bar{n}^{\prime}-n+\bar{n}\right)
\end{aligned}
$$

où nous avons utilisé la relation entre des amplitudes de transition des théories $w_{\alpha}$ et $w_{0}$ (voir la démonstration du lemme).

$\mathrm{Au}$ commencement de ce paragraphe nous avons montré que pour des amplitudes de transition non nulles de la théorie $w_{0}$ le nombre $n^{\prime}-\bar{n}^{\prime}-n+\bar{n}$ est multiple de $m$. Il en résulte que l'intégrale en question est égale à 1 si $m k^{\prime}+n^{\prime}-\bar{n}^{\prime}=m k+n-\bar{n}$ et dans le cas contraire l'intégrale est nulle.

Deux conclusions en résultent. La première est déjà connue: la charge totale se conserve dans l'interaction. La deuxième est plus intéressante: l'amplitude calculée est numériquement égale à l'amplitude correspondante de la théorie $w \equiv w_{0}$.

Nous sommes maintenant en mesure de prouver que la théorie $\bar{w}$ prédit les mêmes probabilités de transition pour les particules que la théorie initiale $w$.

Supposons qu'avant l'interaction un observateur voit $n$ particules et $\bar{n}$ antiparticules dans un état décrit par l'élément $a_{n, \bar{n}}$ de l'algèbre $A$. Calculons la probabilité de trouver après l'interaction $n^{\prime}$ particules et $\bar{n}^{\prime}$ antiparticules dans l'état décrit par $b_{n^{\prime}, \vec{n}^{\prime}} \in A$. 
Dans la théorie initiale cette probabilité est égale à

$$
\left.\left\langle\psi_{w_{0}}^{\text {in }}\left(b_{n^{\prime}, \bar{n}^{\prime}}\right), S_{w_{0}} \psi_{w_{0}}^{\text {in }}\left(a_{n, \bar{n}}\right)\right\rangle\right|^{2} \text {. }
$$

Pour la théorie $\bar{w}$ le calcul est plus compliqué. En voyant des particules l'observateur ne connait pas au-dessus de quel vide ces particules sont excitées. Il peut prendre pour état initial tout vecteur $\psi_{\bar{w}}^{\text {in }}\left(a_{n, \bar{n}} ; f\right)$ à condition que le vide $\psi_{\bar{w}}(1, f)$ soit normé à l'unité. Nous allons voir que le résultat ne dépend pas du choix du vide initial.

Comme l'observateur ne distingue pas des particules excitées audessus des vides différents, la probabilité totale de trouver $n^{\prime}$ particules et $\bar{n}^{\prime}$ antiparticules dans l'état $b_{n^{\prime}, \bar{n}^{\prime}}$ après l'interaction est égale pour lui à la somme

$$
\sum_{k^{\prime}=-\infty}^{\infty}\left|\left\langle\psi_{\bar{w}}^{\text {in }}\left(b_{n^{\prime}, \bar{n}^{\prime}} ; f_{k^{\prime}}\right), S_{\bar{w}} \psi_{\bar{w}}^{\text {in }}\left(a_{n, \bar{n}} ; f\right)\right\rangle\right|^{2} .
$$

Décomposons $f$ :

$$
f=\sum_{k=-\infty}^{\infty} c_{k} f_{k} \quad \text { où } \quad \sum_{k=-\infty}^{\infty}\left|c_{k}\right|^{2}=1
$$

parce que le vide $\psi_{\bar{w}}(1, f)$ est supposé normé à l'unité.

En substituant cette décomposition dans la somme ci-dessus et en utilisant l'expression connue pour l'amplitude de transition entre deux états excités au-dessus des vides de charges fixées on trouve facilement que la somme est égale à

d'où l'assertion.

$$
\left|\left\langle\psi_{w_{0}}^{\text {in }}\left(b_{n^{\prime}, \bar{n}^{\prime}}\right), S_{w_{0}} \psi_{w_{0}}^{\text {in }}\left(a_{n, \bar{n}}\right)\right\rangle\right|^{2} \cdot \sum_{k=-\infty}^{\infty}\left|c_{k}\right|^{2}
$$

On voit donc que les théories $w$ et $\bar{w}$ sont physiquement équivalentes et ne se distinguent que par la façon de décrire la non-conservation de la charge des particules.

En physique on emploie encore une façon de décrire la non-conservation de la charge - c'est le formalisme du spurion. On appelle spurion une particule hypothétique qui ne porte pas d'énergie-impulsion mais possède par exemple la charge (ou d'autres nombres quantiques). Alors si on a un processus avec changement de la charge des particules on dit que ce processus est accompagné d'une création ou d'une destruction de spurions chargés de sorte que la charge totale soit conservée.

Il est clair que le passage de $w$ à $\bar{w}$ est équivalent à l'introduction du spurion de la charge $m$ dans la théorie $w$.

On peut prendre pour opérateurs de création et de destruction du spurion deux opérateurs du commutant $R_{\bar{w}}^{\prime}$ de la représentation réductible $R_{\bar{w}}[5]$ :

où

$$
a_{s p}^{+}=B\left(f_{1}\right) a_{s p}=B\left(f_{-1}\right)
$$

$$
f_{ \pm 1}(\alpha) \equiv \exp ( \pm i m \alpha)
$$


Rappelons que nous désignons par $B(f)$ l'opérateur du commutant $R_{\bar{w}}^{\prime}$ défini par l'équation:

$$
[B(f) x]_{w_{\alpha}}=f(\alpha)[x]_{w_{\alpha}}
$$

où $x$ est un vecteur arbitraire de $H_{\bar{w}},[x]_{w_{\alpha}}$ - sa composante dans $H_{w_{\alpha}}$.

Les opérateurs de création et de destruction du spurion sont unitaires $\left(a_{s p}^{+} a_{s p}=1\right)$ et permutables avec le champ $A_{\bar{w}}(x)$ avec les champs

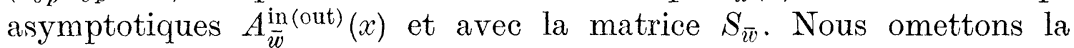
démonstration des assertions ci-dessus.

Par définition:

$$
\begin{aligned}
& a_{s q}^{+} \psi_{\bar{w}}^{\text {in (out) }}\left(a, f_{n}\right)=\psi_{\bar{w}}^{\text {in (out) }}\left(a, f_{n+1}\right) \\
& a_{s p} \psi_{\bar{w}}^{\text {in out) }}\left(a, f_{n}\right)=\psi_{\bar{w}}^{\text {in out) }}\left(a, f_{n-1}\right)
\end{aligned}
$$

d'où on voit que le vide $\psi_{\bar{w}}(1,1)$ est le vecteur cyclique dans $H_{\bar{w}}^{\text {as }}$ pour l'ensemble d'opérateurs composé du champ $A_{\bar{w}}^{\text {in }}(x)$ et des opérateurs de création et de destruction du spurion.

\section{Conclusion}

1. Dans le cas considéré la théorie $\bar{w}$ avec dégénérescence du vide est complèment équivalente à la théorie $w$ dont le vide est non dégénéré. Les deux théories prédisent les mêmes probabilités de transition pour les particules asymptotiques.

2. La symétrie qui se rétablit au passage de $w$ à $\bar{w}$ correspond à une loi de conservation pour le système total composé des particules et du vide.

\section{References}

1. HAAG, R.: Phys. Rev. 112, 669 (1958).

2. Ruglle, D.: Helv. Phys. Acta 35, 147 (1962).

3. Borchers, H. J.: Commun. Math. Phys. 1, 49 (1965).

4. Bourbaki, N.: Eléments de mathématique, Intégration, $2^{\circ}$ éd. chap. 4.

5. VASsilev, A. N.: La structure algébrique de la représentation et le problème de la symétrie pour une classe de fonctionelles de Wightman, soumis à Commun. Math. Phys.

A. N. Vassilev

Leningrad State University

Department of Theoretical Physics

Leningrad, B-164, USSR 